\title{
The role of melanocytes in oral mucosa: From embryologic origin to oral mucosal melanoma: A short review
}

\author{
Ricardo Hsieh ${ }^{1,2 *}$, Raquel Silva ${ }^{2}$ and Silvia Vanessa Lourenço ${ }^{1,2}$ \\ ${ }^{1}$ General Pathology of Department of Stomatology, School of Dentistry, University of São Paulo, Brazil \\ ${ }^{2}$ LIM-06, Institute of Tropical Medicine, University of São Paulo, Brazil
}

\begin{abstract}
Melanoblasts are precursor cells of melanocytes, that arise from neural crest in vertebrates and through several cycles of migration and proliferation, they populate the basal layer of epidermis; hair bulb; eyes; ears and meninges.

However, melanocytes also situate in the basal layer of stratified squamous epithelia that is lining in the mouth. The role of melanocytes in the pathophysiology of the oral mucosa still poor understand, and differently from skin melanocytes, they are in a photo-protected site

Physiologically, oral melanocytes may or may not produce melanin, however non-physiological alterations related to genetic, metabolic, endocrine, chemical and physical factors, and to infections, inflammatory and neoplasic processes could interfere in the oral pigmentation

Primary Oral Mucosal Melanoma (POMM) develops from malignant transformation of melanocytic cell localized in the basal layer of the oral mucosa, which incidence is between 0.2 to $8 \%$ of all melanomas, and representing $0.5 \%$ of all malignant neoplasias of the oral cavity

A better comprehension of the neural crest cells; melanoblasts and melanocytes development and proliferation, and also the melanogenesis and molecular pathways, it could help to understand more about the role of the oral mucosal melanocytes, moreover, and to improve all diagnostic techniques and treatment for Primary Oral Mucosal Melanomas.
\end{abstract}

\section{Background}

In 1868, the Swiss embryologist Wilhelm His identified a distinctive transient embryonic cell population, the neural crest, which has been called as the "fourth germ layer" due to its importance and its capacity of giving rise to various differentiated cells types in adult organisms and it is unique in vertebrates [1].

In neurulation phase of Embryogenesis, the neural tube is formed after fusion of the neural folds, and it is covered by a future epidermal ectoderm, whilst neural crest cells are positioned on its dorsal edges of the forming neural folds. Once the neural tube has closed, the neural crest cells form a transient and not terminally differentiated population of cells. Thus, the interaction between neural plate and the epidermal ectoderm causes the induction of neural crest cells, which colonize extensively throughout the developing embryo to different sites along defined and complex pathways. However, melanoblasts migrate through a pathway that others crest cells cannot, as the path between somite and ectoderm [1-3].

During the migration neural crest cells undergo an epithelialmesenchymal transition phenomenon and differentiate into a very great range of derivatives, such as: melanoblast and melanocytes; neurons and glias; autonomic nerval system; Schwann cells; pericytes and smooth muscles cells of vascular system; adrenal medulla; leptomeninges. Moreover neural crest cells are very important in the faryngeal arches and head, forming bone, cartilage and adipose tissues, as well as eyes, ears and teeth $[1,2,4-7]$. Indeed, the neural crest can generate wide variety of cell and tissue types, hence it represents a multipotent cell population, also known as neural crest stem cells [1].
In mouse model, neural crest cells undergo two migration pathways: ventral pathway, they migrate in the space between the somites and the neural tube and giving rise to neuronal and glial cells; Schwann cells and chromaffin cells in the adrenal medulla, whereas, dorsolateral pathway they migrate between the somite and the nonneural ectoderm and differentiating in melanoblast and melanocytes $[5,6]$. In the trunk, the neural crest cells of all lineages migrate first into the migration staging area (space located between the dorsal part of the somite, the lateral part of the neural tube and the ventral part of the ectoderm), where they receive signals directing their migration and specification. The expression of KIT (tyrosine kinase receptor) in the neural crest cells has been pointed as determinant in this melanogenic fate. Besides, the importance of Wnt signaling, through activation of $\beta$-catenin, also has been suggested and proven by studies in fish [6].

\section{Melanoblast and melanocytes: Oral mucosa}

Pigment cells are one derivative of the neural crest, in contrast to ectotherms, birds and mammals have a single type of skin pigment cell, the melanin-containing melanocyte [2]. Melanoblasts are precursor

${ }^{\star}$ Correspondence to: Ricardo Hsieh, General Pathology of Department of Stomatology, School of Dentistry, University of São Paulo, Brazil, E-mail: r.hsieh@usp.br

Key words: oral mucosa, POMM

Received: February 09, 2020; Accepted: February 21, 2020; Published: February 25,2020 
cells of melanocytes, that arise from neural crest in vertebrates and through several cycles of migration and proliferation, they populate the basal layer of epidermis; hair bulb; eyes; ears and meninges $[6,8]$.

Some follicular melanoblasts concentrate in the niche of the hair follicle, known as bulge, and they form the melanocytes stem cells (MSC) with the responsibility to maintain homeostasis. However, other follicular melanoblast migrate towards the bulb of the hair follicle, where they differentiate into mature melanocytes, which have capacity to produce melanin in specialized organelles, called melanosomes $[5,9,10]$.

To protect our body against hazardous ultraviolet radiation from sun light, the melanocyte contains melanossome, an unique melanin producer organelle to provide photo-protection [10]. However, melanocytes also situate in the basal layer of stratified squamous epithelia that is lining in the mouth. The role of melanocytes in the pathophysiology of the oral mucosa still poor understand, and differently from skin melanocytes, they are in a photo-protected site [11].

It is known that the number of melanocytes should be the same in the oral epithelium, however the variety of the oral mucosa colour depend on melanogenic activity of the melanocytes in the basal layer of the epithelium and difference in quantity and quality of melanin and melanossomes. Physiologically, oral melanocytes may or may not produce melanin, however non-physiological alterations related to genetic, metabolic, endocrine, chemical and physical factors, and to infections, inflammatory and neoplasic processes could interfere in the oral pigmentation [12] (Figure 1).

\section{Primary oral mucosal melanoma}

Malignant Melanoma represents a malignancy showing evidence of melanocytic differentiation that can be identified histopathologically or ultrastructurally [13], it is characterized by a high tendency to metastasize and striking resistance to conventional therapies other than surgery [14]. According to American Cancer Society about 100,350 new cases of melanoma will be diagnosed in 2020 , and 6,850 people are expected to die of melanoma (www.cancer.org).

Approximately $1-2 \%$ of all melanomas originate from the mucosal membranes in the digestive, respiratory and genitourinary tracts $[15,16]$. Primary Oral Mucosal Melanoma (POMM) develops from malignant transformation of melanocytic cell localized in the basal layer of the oral mucosa, which incidence is between 0.2 to $8 \%$ of all melanomas and representing $0.5 \%$ of all malignant neoplasias of the oral cavity [17-19].

Epidemiologically, POMM is more prevalent among Asians, Africans and Mixed Population than Whites. There is a peak of incidence between 40 to 60 years of age, and the gender ratio has a slightly preference for male than female, approximately $2: 1$. The most preferred sites are: hard palate; alveolar crest; labial and buccal mucosa [12,17,20,21] (Figure 2).

Clinically, POMM usually present: a melanotic pigmentation, however up to $20 \%$ is amelanotic lesions, pain, bleeding and ulceration. Most common sites of metastasis include lymph nodes, lung, bone, liver and brain. At the same time it has a poor prognosis comparing to its cutaneous counterpart, with 5 -year survival rate of $5 \%$ to $20 \%$ $[12,17,20,21]$.

Histopatologically, the Western Society of Teachers of Oral Pathology (Westop) agreed that POMM should be classified separately from the cutaneous forms in 1995. In 2002, Prasad et al. proposed to

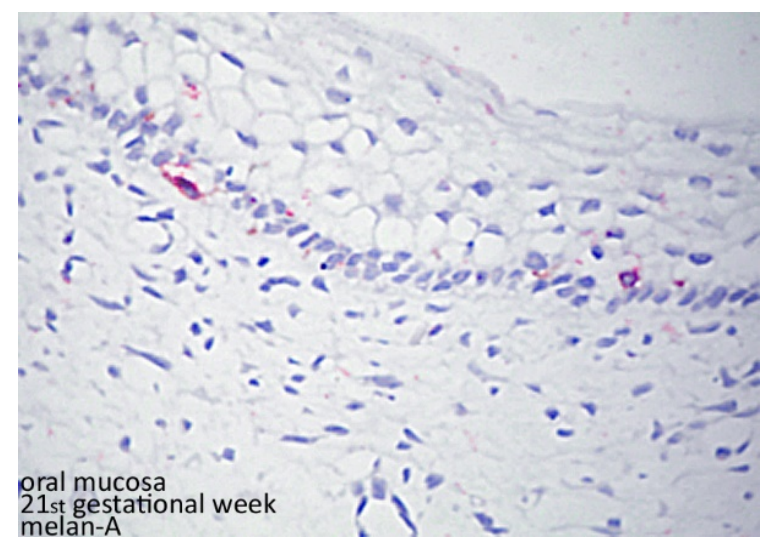

Figure 1. Histopatological figure: melanocytes in the basal layer of the epithelium identified by Melan-A primary antibody and reveled by permanent red chromogen of human fetal oral mucosa

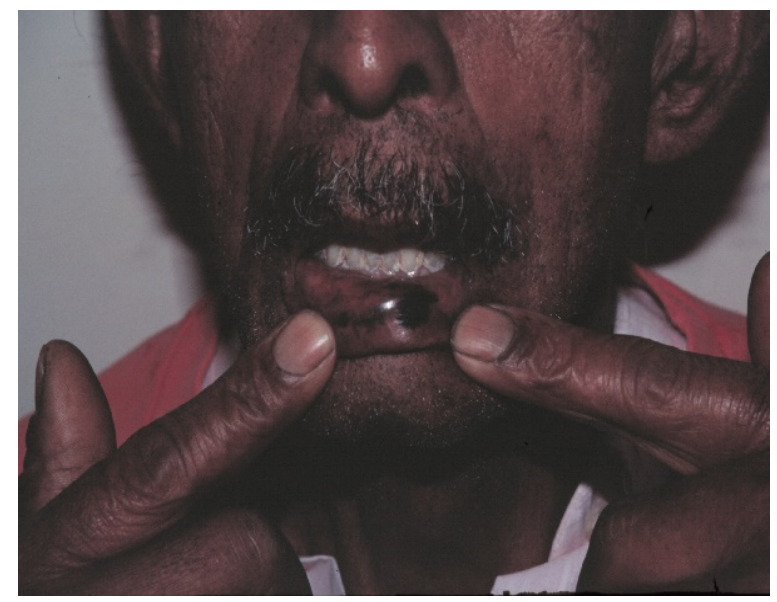

Figure 2. Melanocytic lesion in the lower lip - primary oral mucosal melanoma

classify tumor limited in the oral cavity into three levels: level 1 (in situ tumor); level 2 (invasion in the lamina propria); level 3 (deeply invasion to bone and muscle) $[22,23]$.

The aetiology of POMM still poorly understood. A definitely precursor lesion has not been identified yet, but atypical melanocytic hyperplasia may represent a proliferative phase before the tumorigenesis. No evidence of mechanical trauma, tobacco smoking, chronic inflammation process, HPV infection, alcohol or other chemical product exposure plays any role in its pathogenesis, however they could be considered as possible predisposing factors [12,21]. Meantime, some intracellular molecular signaling pathway have been identified in oral mucosal melanogenesis, as dysregulation of cell cycle progression; apoptosis; cell-to-cell interactions; Ckit pathway; MCR1 polymorphism could play role in the POMM etiopathogenesis [12]

\section{POMM treatment}

Surgical resection of the lesions with negative surgical margins, and chemo and radiotherapy as adjuvant treatment, additionally, introduction of immune stimulating antibodies and signal transduction inhibitors may improve the outcome of POMM metastasis [16,21].

\section{Conclusion}

A better comprehension of the neural crest cells; melanoblasts and melanocytes development and proliferation, and also the melanogenesis and molecular pathways, it could help to understand 
more about the role of the oral mucosal melanocytes, moreover, and to improve all diagnostic techniques and treatment for Primary Oral Mucosal Melanomas.

\section{References}

1. La Noce M, Mele L, Tirino V, Paino F, De Rosa A, et al. (2014) Neural crest stem cell population in craniomaxillofacial development and tissue repair. Eur Cells Mater 28: 348-357.

2. Parichy DM, Reedy MV, Erickson CA (2013) Regulation of melanoblast migration and differentiation In: Intergovernmental panel on climate change. [Ed]. The pigmentary system. Oxford, UK: Blackwell publishing ltd pp: 108-39.

3. Tosney KW (2004) Long-distance cue from emerging dermis stimulates neural crest melanoblast migration. Dev Dyn 229: 99-108.

4. Thomas AJ, Erickson CA (2008) The making of a melanocyte: the specification of melanoblasts from the neural crest. Pigment Cell Melanoma Res 21: 598-610. [Crossref]

5. Larue L, De Vuyst F, Delmas V (2013) Modeling melanoblast development. Cell Mol Life Sci 70: 1067-1079.

6. Li A (2014) The biology of melanocyte and melanocyte stem cell. Acta Biochim Biophys Sin (Shanghai) 46: 255-260. [Crossref]

7. Yamaguchi Y, Hearing VJ (2014) Melanocytes and their diseases. Cold Spring Harb Perspect Med 4.

8. Liu J, Fukunaga-Kalabis M, Li L, Herlyn M (2014) Developmental pathways activated in melanocytes and melanoma. Arch Biochem Biophys 563: 13-21.

9. Hirobe T (2011) How are proliferation and differentiation of melanocytes regulated? Pigment Cell Melanoma Res 24: 462-478. [Crossref]

10. Lang D, Mascarenhas JB, Shea CR (2013) Melanocytes, melanocyte stem cells, and melanoma stem cells. Clin Dermatol 31: 166-178.

11. Barrett AW, Raja AM (1997 The immunohistochemical identification of human oral mucosal melanocytes. Arch Oral Biol 42: 77-81. [Crossref]

12. Feller L, Masilana A, Khammissa RA, Altini M, Jadwat Y, et al. (2014) Melanin: the biophysiology of oral melanocytes and physiological oral pigmentation. Head Face Med 10: 8. [Crossref]
13. Kim J, Lazar AJ, Davies MA, Homsi J, Papadopoulos NE, et al. (2012) BRAF, NRAS and KIT sequencing analysis of spindle cell melanoma. J Cutan Pathol 39: 821-825. [Crossref]

14. Colombino M, Paliogiannis P, Cossu A, De Re V, Miolo G, et al. (2019) BRAF Mutations and Dysregulation of the MAP Kinase Pathway Associated to Sinonasal Mucosal Melanomas. J Clin Med 8: 1577.

15. Zebary A, Jangard M, Omholt K, Ragnarsson-Olding B, Hansson J (2013) KIT, NRAS and BRAF mutations in sinonasal mucosal melanoma: A study of 56 cases. $\mathrm{Br}$ J Cancer 109: 559-64.

16. Soma PF, Pettinato A, Agnone AM, Donia C, Improta G, et al. (2014) Oral malignant melanoma: A report of two cases with BRAF molecular analysis. Oncol Lett 8: 12831286.

17. Lourenēo SV, A MS, Sotto MN, Bologna SB, Giacomo TB, et al. (2009) Primary oral mucosal melanoma: a series of 35 new cases from South America. Am J Dermatopathol 31: 323-330. [Crossref]

18. Hsieh R, Nico MMS, Coutinho-Camillo CM, Buim ME, Sangueza M, et al. (2013) The CDKN2A and MAP kinase pathways: Molecular roads to primary oral mucosal melanoma. Am J Dermatopathol 35: 1-10.

19. Hsieh R, Nico MMS, Camillo CMC, Oliveira KK, Sangueza M, et al. (2017) Mutational status of NRAS and BRAF genes and protein expression analysis in a series of primary oral mucosal melanoma. Am J Dermatopathol 39: 1-10.

20. Garzino-Demo P, Fasolis M, Maggiore GM, Pagano M, Berrone S (2004) Ora mucosal melanoma: a series of case reports. J Craniomaxillofac Surg 32: 251-257. [Crossref]

21. Vijay Kumar, Vishnoi JR, Sameer Gupta, Sanjeev Misra (2015) Primary malignan melanoma of oral cavity: A tertiary care center experience. Natl J Maxillofac Surg 6: 167-71.

22. Aguas SC, Quarracino MC, Lence AN, Lanfranchi-Tizeira HE (2009) Primary melanoma of the oral cavity: ten cases and review of 177 cases from literature. Med Oral Patol Oral Cir Bucal 14: 265-271. [Crossref]

23. Patel SG, Prasad ML, Escrig M, Singh B, Shaha AR, et al. (2002) Primary mucosal malignant melanoma of the head and neck. Head Neck 24: 247-257.

Copyright: (C2020 Hsieh R. This is an open-access article distributed under the terms of the Creative Commons Attribution License, which permits unrestricted use, distribution, and reproduction in any medium, provided the original author and source are credited. 